PHYSICAL REVIEW D 95, 115006 (2017)

\title{
Electroweak baryogenesis from a dark sector
}

\author{
James M. Cline* \\ CERN, Theoretical Physics Department, 1211 Geneva 23, Switzerland \\ and Department of Physics, McGill University, 3600 Rue University, Montréal, Québec H3A 2T8, Canada \\ Kimmo Kainulainen ${ }^{\dagger}$ \\ Department of Physics, University of Jyväskylä, P.O. Box 35 (YFL), FIN-40014 Jyväskylä, Finland \\ and Helsinki Institute of Physics, University of Helsinki, P.O. Box 64, FIN-00014 Helsinki, Finland \\ David Tucker-Smith \\ Department of Physics, Williams College, Williamstown, Massachusetts 01267, USA
}

(Received 10 March 2017; published 7 June 2017)

\begin{abstract}
Adding an extra singlet scalar $S$ to the Higgs sector can provide a barrier at tree level between a false vacuum with restored electroweak symmetry and the true one. This has been demonstrated to readily give a strong phase transition as required for electroweak baryogenesis. We show that with the addition of a fermionic dark matter particle $\chi$ coupling to $S$, a simple UV-complete model can realize successful electroweak baryogenesis. The dark matter gets a $C P$ asymmetry that is transferred to the standard model through a $C P$ portal interaction, which we take to be a coupling of $\chi$ to $\tau$ leptons and an inert Higgs doublet. The $C P$ asymmetry induced in left-handed $\tau$ leptons biases sphalerons to produce the baryon asymmetry. The model has promising discovery potential at the LHC, while robustly providing a large enough baryon asymmetry and correct dark matter relic density with reasonable values of the couplings.
\end{abstract}

DOI: 10.1103/PhysRevD.95.115006

\section{INTRODUCTION}

Electroweak baryogensis (EWBG) is the most experimentally testable mechanism for explaining the baryon asymmetry of the Universe (BAU), and as such it is coming under increasing pressure from LHC constraints on new physics below the $\mathrm{TeV}$ scale. The minimal supersymmetric standard model has practically been excluded for realizing EWBG [1-3], while the remaining parameter space for two Higgs doublet models to do so is increasingly narrow [4-6] (see however [7]). The challenges are intricate since not only must one provide new particles coupling to the Higgs field in order to make the electroweak phase transition strongly first order, but also large $C P$ violation to get sufficient baryon production. Constraints on electric dipole moments often restrict such new sources of $C P$ violation.

Adding an extra scalar singlet field to these models alleviates the tension [8-12]. One way in which the singlet can help is by providing a tree-level barrier that gives a robust way of making the phase transition more strongly first order [13-15], which has been exploited in recent work

\footnotetext{
*jcline@physics.mcgill.ca

kimmo.kainulainen@jyu.fi

dtuckers@williams.edu
}

Published by the American Physical Society under the terms of the Creative Commons Attribution 4.0 International license. Further distribution of this work must maintain attribution to the author(s) and the published article's title, journal citation, and DOI. on EWBG [16-24]. Another feature of the singlet-assisted transition is that it can lead to observable gravitational waves [7,24-31] (see also [32]).

Many of the baryogenesis studies have relied upon dimension-5 or -6 couplings of the scalar to standard model (SM) fermions in order to get $C P$-violating interactions in the bubble walls during the phase transition. The inverse mass scale of the nonrenormalizable operator must be relatively small, indicating the need for additional new physics just beyond the $\mathrm{TeV}$ scale. It would be more satisfying to have an ultraviolet-complete picture involving only renormalizable interactions.

In this paper we introduce a new realization of EWBG that meets these criteria. It relies upon a Majorana fermion $\chi$ that can couple to the scalar via $S \bar{\chi} \gamma_{5} \chi$. A $Z_{2}$ symmetry is imposed so that $\chi$ can couple to standard model leptons, for example the $\tau$ lepton doublet $L_{\tau}$, in combination with an inert Higgs doublet $\phi$, through the interaction $y \bar{L}_{\tau} \phi \chi$. We dub this a " $C P$ portal interaction," since its purpose is to transmit the $C P$ asymmetry between the two helicity states of $\chi$, that is, generated at the bubble wall during the firstorder electroweak phase transition, to $L_{\tau}$ by (inverse) decays of $\phi$. An interesting feature of the model is that $\chi$ is a dark matter candidate if $m_{\chi}<m_{\phi}$ (otherwise $\phi$ is the dark matter), whose relic density is determined by the same coupling $y$ as enters into the baryon asymmetry.

Numerous previous works have explored possible links between electroweak baryogenesis and dark matter $[20,29,33-41]$. In the case where the scalar singlet is the 
dark matter candidate, it must be subdominant to the main dark matter (DM) constituent if it is to provide a strong enough phase transition for baryogenesis (while it may nevertheless be directly detectable) [16]. Unlike previous works, here the dark matter plays an essential role in generating the baryon asymmetry rather than strengthening the phase transition. Moreover we show it can be accomplished without any explicit $C P$ violation in the dark sector, since $C P$ can be violated spontaneously in the DM interactions with the scalar singlet in the bubble wall, where $S$ gets a vacuum expectation value (VEV). Since the VEV disappears at low temperatures and $C P$ is restored, the model is in this case immune to constraints from electric dipole moment searches.

We account for an issue that can be important in models with a tree-level barrier, namely the propensity for supercooling during the phase transition, especially if it is strongly first order. It is necessary to construct the bubble wall solutions at temperatures below the often considered critical temperature $T_{c}$, at which bubble nucleation actually becomes faster than the Hubble rate. We find that moderate supercooling is required, with nucleation temperatures $T_{n}$ typically 20\%-30\% lower than $T_{c}$. However $T_{n} \sim 100 \mathrm{GeV}$, and the new particles are not too Boltzmann suppressed to yield efficient baryogenesis.

\section{MODEL AND MECHANISM}

For simplicity, we study the phase transition in a limit of the scalar potential that has a $Z_{2}$ symmetry under $S \rightarrow-S$,

$V_{0}=\lambda_{h}\left(|H|^{2}-\frac{1}{2} v_{0}^{2}\right)^{2}+\frac{1}{4} \lambda_{S}\left(S^{2}-w_{0}^{2}\right)^{2}+\frac{1}{2} \lambda_{m}|H|^{2} S^{2}$.

Eventually one would like to consider models in which this restriction is removed, since the couplings of $S$ to fermions do not generically respect $S \rightarrow-S$ symmetry. However it will be seen that $Z_{2}$ symmetry is preserved in the finitetemperature scalar potential when the coupling of $S$ to new fermions takes the form $i S \bar{\chi} \gamma_{5} \chi$, appropriate in the case where $S$ is interpreted as a pseudoscalar.

In addition to the singlet scalar, we introduce a singlet Majorana fermion $\chi$ and an inert doublet $\phi$ with couplings

$$
\frac{1}{2} \bar{\chi}\left(\left(\eta P_{R}+\eta^{*} P_{L}\right) S+m_{\chi}\right) \chi+y \bar{L}_{\tau} \phi P_{R} \chi+\text { H.c. }
$$

The hypercharge of $\phi$ is equal to that of left-handed SM leptons. For simplicity we have coupled $\phi \chi$ only to the $\tau$ lepton doublet. In general it could be a linear combination of lepton flavors, but with a framework like minimal flavor violation in mind, this could be a good approximation to the case where the coupling to $L_{\tau}$ dominates. In such a more complete setup, constraints from lepton flavor violating decays such as $\mu \rightarrow e \gamma$ induced at one loop by $\phi$ exchange will give limits on the additional couplings.

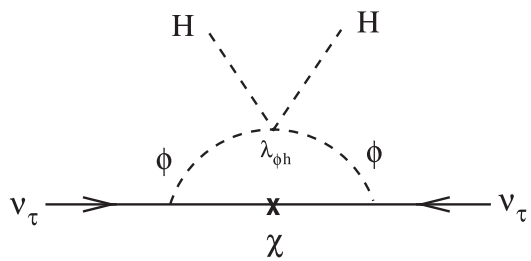

FIG. 1. Loop contribution to Weinberg operator $\sim\left(\lambda_{\phi h} y^{2} m_{\chi} / 16 \pi^{2} m_{\phi}^{2}\right)\left(L_{\tau} H\right)^{2}$ constrained by $\nu_{\tau}$ mass.

The mass $m_{\chi}$ is taken to be real and the coupling $\eta$ complex, so that in general there is $C P$ violation via their relative phase. The field-dependent mass has a spatially varying phase $\theta(z)$ in the bubble walls of the phase transition, if $S(z)$ has a nontrivial profile:

$$
\theta=\arg \left(\eta S+m_{\chi}\right)=\tan ^{-1}\left(\frac{|\eta| S}{m_{\chi}}\right),
$$

where in the last expression we have assumed maximal phase, $\eta=i|\eta|$, which simplifies the effective scalar potential to be discussed below. The coordinate $z$ denotes distance transverse to the wall, with $z<0$ corresponding to the interior where electroweak symmetry is broken, and $z>0$ the symmetric phase outside of the bubble.

We will show that a baryon asymmetry can be efficiently generated by a $C P$ asymmetry between the two helicity components of $\chi$ that is produced at the bubble wall and subsequently gets transmitted to $L_{\tau}$ leptons by the inverse decay $\chi L_{\tau} \rightarrow \phi$. Sphalerons are sourced by the $L_{\tau}$ chemical potential to produce the baryon asymmetry.

For simplicity we have neglected the possible couplings of $\phi$ to the SM Higgs doublet in the scalar potential. In particular, the interaction $\lambda_{\phi h}\left(\phi^{\dagger} H\right)^{2}$ violates lepton number, since it is necessary to assign $\phi$ lepton number 1 in (2), and consequently generates an unacceptably large Majorana mass for $\nu_{\tau}$ at one loop, as shown in Fig. 1. We impose lepton number as an additional approximate symmetry to forbid such a coupling.

\section{EFFECTIVE POTENTIAL AND PHASE TRANSITION}

We follow Refs. [14,15], starting from the tree-level potential for the Higgs doublet $H$ and real singlet $S$ (1). Parameters can be chosen such that the $Z_{2}$ symmetry under $S \rightarrow-S$ breaks spontaneously at high temperatures, giving $S$ a VEV (with $H=0$ ) in the electroweak symmetric vacuum, while the true vacuum is along the $H$ axis at $T=0$. The finite-temperature effective potential for the real fields $H=h / \sqrt{2}$ and $S$ can be written in the form

$$
\begin{aligned}
V= & \frac{\lambda_{h}}{4}\left(h^{2}-v_{c}^{2}+\frac{v_{c}^{2}}{w_{c}^{2}} S^{2}\right)^{2}+\frac{\kappa}{4} S^{2} h^{2} \\
& +\frac{1}{2}\left(T^{2}-T_{c}^{2}\right)\left(c_{h} h^{2}+c_{s} S^{2}\right)+\frac{1}{12} T^{2} \operatorname{Re}\left(\eta m_{\chi}\right) S
\end{aligned}
$$


where the parameter $w_{0}$ has been traded for its counterpart $w_{c}$ at the critical temperature of the phase transition $T_{c}, v_{c}$ is the corresponding critical VEV of $h$, and the following relations hold:

$$
\begin{gathered}
\kappa \equiv \lambda_{m}-2 \lambda_{h} \frac{v_{c}^{2}}{w_{c}^{2}}, \\
T_{c}^{2}=\frac{\lambda_{h}}{c_{h}}\left(v_{0}^{2}-v_{c}^{2}\right) .
\end{gathered}
$$

Here the coefficients $c_{h}$ and $c_{s}$ encode the $O\left(T^{2}\right)$ corrections to the masses of $h$ and $S$, and are given in terms of the gauge and other couplings by

$$
\begin{aligned}
& c_{h}=\frac{1}{48}\left(9 g^{2}+3 g^{2}+12 y_{t}^{2}+24 \lambda_{h}+2 \lambda_{m}\right), \\
& c_{s}=\frac{1}{12}\left(3 \lambda_{h} \frac{v_{c}^{4}}{w_{c}^{4}}+2 \lambda_{m}+|\eta|^{2}\right),
\end{aligned}
$$

where we ignored all SM Yukawa couplings apart from that of the top quark, as well as possible couplings of the inert doublet $\phi$ to the SM Higgs. The zero-temperature masses are given by

$$
\begin{gathered}
m_{h}^{2}=2 \lambda_{h} v_{0}^{2}, \\
m_{s}^{2}=\frac{1}{2} \kappa v_{0}^{2}+\lambda_{h}\left(v_{0}^{2}-v_{c}^{2}\right)\left(\frac{v_{c}^{2}}{w_{c}^{2}}-\frac{c_{s}}{c_{h}}\right) .
\end{gathered}
$$

The actual bubble nucleation temperature $T_{n}$ is lower than the critical temperature $T_{c}$; it is determined by the Euclidean action $S_{3}$ of the bubble solution,

$$
S_{3}=4 \pi \int_{0}^{\infty} d r r^{2}\left(\frac{1}{2}\left(h^{\prime 2}+s^{\prime 2}\right)+V(h, s)-V\left(0, w_{T}\right)\right)
$$

$\left[V\left(0, w_{T}\right)\right.$ being the value of the potential in the false minimum and primes denoting $d / d r]$, through the relation

$$
\exp \left(-S_{3} / T_{n}\right)=\frac{3}{4 \pi} \frac{\left(H\left(T_{n}\right)^{4}\right.}{\left.T_{n}\right)}\left(\frac{2 \pi T_{n}}{S_{3}}\right)^{3 / 2}
$$

where $H$ is the Hubble rate.

To compute the bubble action $S_{3}$, we discretized the spherically symmetric equations of motion for $(h, s)$ following from (10) and solved them using relaxation, subject to the boundary conditions $d h / d r=d s / d r=0$ at $r=0$ and $(h, s)$ approaching the false minimum of the potential as $r \rightarrow \infty$. The solutions turn out to be well described by the thin-wall approximation [42] suitably modified to accommodate two fields, as we describe in Appendix A. This approximate method is numerically much faster than the exact solution, making it useful for scanning over models. As a further check, we recomputed the nucleation temperature $T_{n}$ and VEV $v_{n}$ for models from our random scan using the COSMOTRANSITIONS package [43], verifying consistency with the results from our own code. ${ }^{1}$

Having determined the nucleation temperature and VEV, we demand that $v_{n} / T_{n}>1.1$ to prevent washout of the baryon asymmetry in the broken phase inside the bubble walls $[45,46]$. Finding the correct bubble wall profile is a very complicated problem that essentially depends on the friction exerted on the wall by the nonequilibrium plasma. Here we adopt the following simple approximation. We fix the path in field space by minimizing the potential $V(h, s)$ along radial directions: $\left(\partial_{\rho} V\right)_{\theta} \equiv 0$ and setting $h(\theta) \equiv$ $\rho(\theta) \cos \theta$ and $s(\theta) \equiv \rho(\theta) \sin \theta$. After this we fix the main spatial dependence of the profile by setting

$$
h(z)=\frac{1}{2} v_{n}\left(1-\tanh \left(z / L_{w}\right)\right),
$$

where the wall thickness is estimated as [15]

$$
L_{w} \cong\left[\frac{2.7}{\kappa} \frac{v_{c}^{2}+w_{c}^{2}}{v_{c}^{2} w_{c}^{2}}\left(1+\frac{\kappa w_{c}^{2}}{4 \lambda_{h} v_{c}^{2}}\right)\right]^{1 / 2} .
$$

Here we use the thickness of the one-dimensional solution corresponding to the steady-state phase of the expanding bubble wall, rather than the thickness of the initial critical bubble determined in [44]. The former is thinner than the latter, but still $\gtrsim 6 / T$, justifying the semiclassical approach to computing the baryon asymmetry that we employ below. An example of the wall path in field space, and its spatial profile, is shown in Fig. 2.

To maintain consistency with our adoption of the $Z_{2}$ symmetric tree-level potential, we can set $\operatorname{Re}\left(\eta m_{\chi}\right)=0$. This corresponds to taking a $C P$-conserving coupling of $S$ to $\chi$, where $S$ transforms as a pseudoscalar. In a realistic treatment, there should be some breaking of the $Z_{2}$ symmetry; otherwise equal and opposite baryon asymmetries are produced from neighboring regions of the Universe starting from false vacua with $S>0$ or $S<0$, leading to a net vanishing asymmetry. However a small breaking to remove the degeneracy is sufficient to dilute away any regions in the higher-energy vacuum by the evolution of domain walls, and should not change our estimate of the baryon asymmetry in a significant way, as long as the domain walls formed when $S$

\footnotetext{
${ }^{1}$ Before carrying out this more exact analysis, we initially used the results of Ref. [44], which presents semianalytic formulas for the bubble wall profile and action. These formulas are only tractable at the lowest order in an expansion that should converge to the accurate action and tunneling path in field space. We found that this lowest order approximation typically gives a $S_{3} / T$ much larger than the true value, by factors of 10 or more, leading to an underestimate of $T_{n}$ and an overestimate of the strength of the phase transition, $v_{n} / T_{n}$.
} 


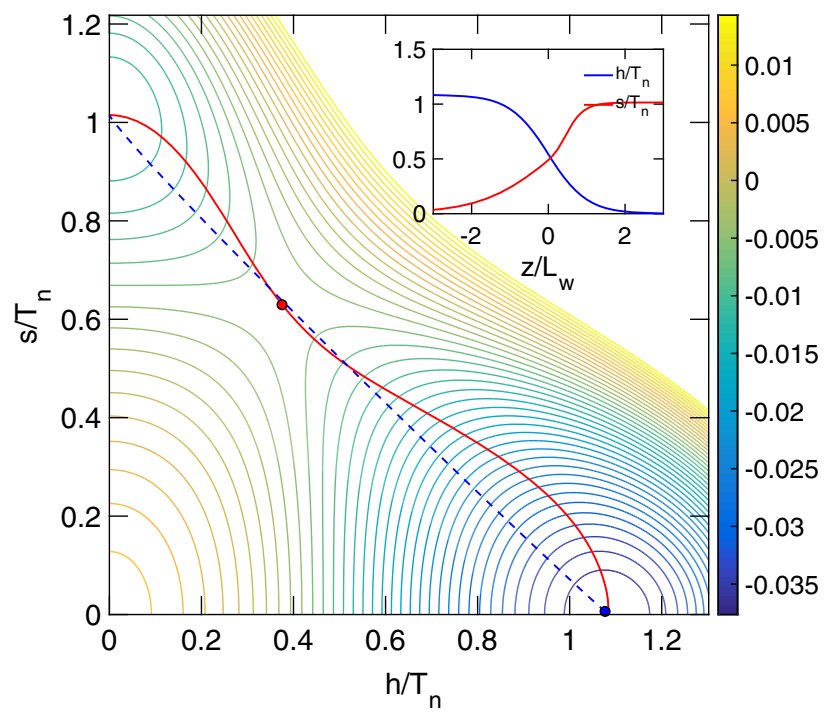

FIG. 2. Example of a path (for the fiducial model of Table I) over the phase transition wall in the $(h, s)$ space (continuous red curve) with the equipotential surfaces included for $V(h, s) / T_{n}^{4}$. The red dot indicates the position of the saddle point. The amplitudes of the fields as a function of $z$ are shown in the inset. Also shown is the tunneling path of the nucleation bubble at $T=T_{n}$ (blue dashed curve).

condenses have time to move away before tunneling to the electroweak symmetry breaking vacuum occurs.

\section{BARYON ASYMMETRY}

To compute the chemical potentials that induce baryon violation, we use the first-order diffusion equations for the chemical potential $\mu_{i}$ and velocity perturbations $u_{i}$ for $i=\chi$, $\phi, L_{\tau},{ }^{2}$ following the method introduced for the minimal supersymmetric standard model (MSSM) in Ref. [47], which was refined by [48]. Here $\mu_{\chi}$ is the potential for negative minus positive helicity $\chi$ 's. In the limit $m_{\chi} \rightarrow 0$, the positive and negative helicity states would correspond to the particle and antiparticle states of the massless fermion. With $m_{\chi}>0$, there is no distinction between particle and antiparticle, but there is still approximate conservation of the helicities, whose damping by mass effects will be taken into account in the Boltzmann equations. Following the notation of Ref. [48], the ensuing fluid equations can be concisely written as

$$
\begin{aligned}
& A_{\chi}\left(\begin{array}{c}
\mu_{\chi}^{\prime} \\
u_{\chi}^{\prime}
\end{array}\right)=C_{\chi}-v_{w} m_{\chi}^{2 \prime}\left(\begin{array}{c}
K_{2, \chi} \mu_{\chi} \\
K_{6, \chi} u_{\chi}
\end{array}\right)+\left(\begin{array}{c}
0 \\
S_{\chi}^{h}
\end{array}\right), \\
& A_{i}\left(\begin{array}{c}
\mu_{i}^{\prime} \\
u_{i}^{\prime}
\end{array}\right)=C_{i}, \quad \text { for } i=\phi, \tau,
\end{aligned}
$$

\footnotetext{
${ }^{2} u_{L}$ has dimensions of mass, and is proportional to the actual velocity perturbation.
}

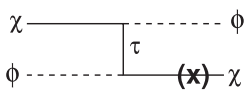

(a)

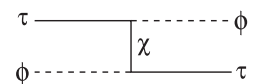

(b)

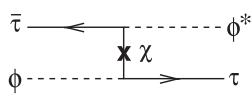

(c)
FIG. 3. Dominant infrared-sensitive processes governing elastic and helicity-flipping scattering rates. The lines marked with $(\mathbf{x})$ in (a) and (c) indicate possible helicity flips of the $\chi$ that enter into the rate $\Gamma_{h f}$, where as (b) is helicity conserving. These are computed in the electroweak symmetric phase in front of the wall, neglecting hypercharge interactions.

where the coefficient matrices $A_{i}$ and the collision factors $C_{i}$ are given by

$A_{i} \equiv\left(\begin{array}{cc}v_{w} K_{1, i} & 1 \\ -K_{4, i} & v_{w} K_{5, i}\end{array}\right), \quad C_{i} \equiv\left(\begin{array}{c}C_{i}^{\mu} \\ -\tilde{\Gamma}_{\mathrm{el}, \mathrm{i}} u_{i}\end{array}\right)$,

with

$$
\begin{aligned}
C_{\chi}^{\mu} & =2 \tilde{\Gamma}_{\mathrm{hf}} \mu_{\chi}+2 \tilde{\Gamma}_{d}\left(\mu_{\chi}+c \mu_{\tau}-c \mu_{\phi}\right), \\
C_{\phi}^{\mu} & =\tilde{\Gamma}_{d}\left(\mu_{\phi}-\mu_{\tau}-c \mu_{\chi}\right)+2 \tilde{\Gamma}_{\times, \phi}\left(\mu_{\phi}-\mu_{\tau}\right), \\
C_{\tau}^{\mu} & =\tilde{\Gamma}_{d}\left(\mu_{\tau}+c \mu_{\chi}-\mu_{\phi}\right)+2 \tilde{\Gamma}_{\times, \tau}\left(\mu_{\tau}-\mu_{\phi}\right) .
\end{aligned}
$$

Here primes denote $d / d z$, where $z$ is the direction transverse to the bubble wall, $v_{w}$ is the wall velocity, $m_{\chi}=$ $\left(m_{\chi}^{2}+|\eta|^{2} S^{2}\right)^{1 / 2}$ is the magnitude of the field-dependent $\chi$ mass, and the thermal functions $K_{i, j} \equiv K_{i}\left(m_{j} / T\right)$ are defined in [48].

The reaction rates appearing in (14) depend primarily upon the (inverse) decay rates for $\phi \rightarrow L_{\tau} \chi$ and on the scattering processes shown in Fig. 3. These are the dominant reactions because of infrared enhancement when the intermediate particle exchanged in the $t$ channel goes on shell, as described in Appendixes B and C. The tildes denote a particular normalization of the rates [see Eq. (D7) and following Eq. (E5)] that is convenient for verifying conserved quantities, in the present case total lepton number $n_{\phi}+n_{\tau}$, which is conserved by the $\bar{L}_{\tau} \phi \chi$ interaction. $\tilde{\Gamma}_{\mathrm{hf}}$ is the rate of helicity-flipping scatterings due to the diagram of Fig. 3(a) with the $\chi$ mass insertion. $\tilde{\Gamma}_{\times}$is the rate of $\phi \bar{L}_{\tau} \rightarrow \phi^{*} L_{\tau}$ scatterings via mass insertion of the internal $\chi$ shown in Fig. 3(c). $\tilde{\Gamma}_{\mathrm{el}, i}$ is the elastic scattering rate for particle $i$ and $\tilde{\Gamma}_{d}$ is the (inverse) decay rate $\phi \rightarrow L_{\tau} \chi$. Details of the computations of the elastic and helicity-flipping rates are given in Appendixes B and C.

The deviation of the coefficient $c$ from unity quantifies the probability of helicity reversal of $\chi$ in a decay process due to its thermal motion. It is crucial for the mechanism that $c>0$ since otherwise there would be no net production of $\mu_{\tau}$ from the (inverse) decays, as needed to bias the sphalerons. However as discussed in Appendix D, we are far from the regime where $c$ would be small enough to significantly suppress the baryon asymmetry. 


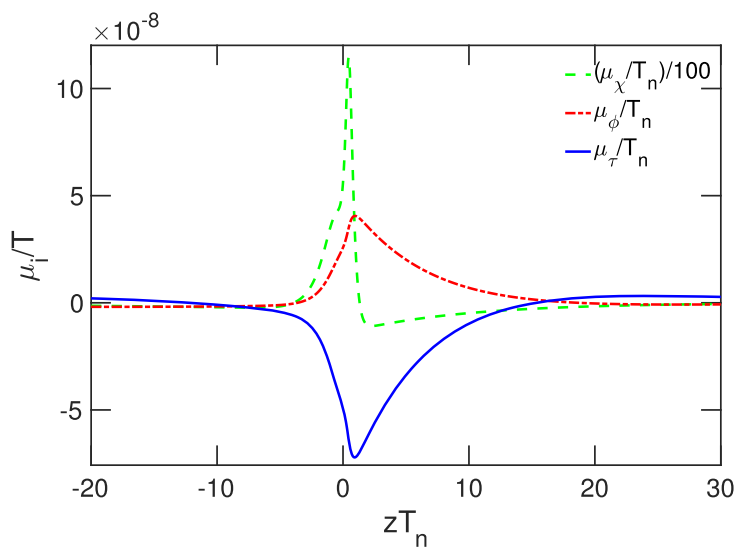

FIG. 4. Solution to diffusion equations for our benchmark model defined in Table I.

Finally, $S_{\chi}^{h}$ is the semiclassical source, which was first derived in $[47,49]$ in the $1 \mathrm{D}$ case and in [50] for 3D. However, these works give the source in a particular spineigenstate basis, while here we find it more convenient to work in terms of helicity eigenstates, defined in the wall rest frame. We derive the correct source in the helicity basis in Appendix E. The result is

$$
S_{\chi}^{h}=v_{w}\left(-K_{8}^{h}\left(m_{\chi}^{2} \theta^{\prime}\right)^{\prime}+K_{9}^{h} \theta^{\prime} m_{\chi}^{2} m_{\chi}^{2 \prime}\right),
$$

where the thermal integrals $K_{8,9}^{h}\left(m_{\chi} / T\right)$ are given in Appendix E. An example of a solution of the fluid equations (14) for the chemical potentials is shown in Fig. 4.

Once $\mu_{\tau}$ is known, the baryon-to-entropy ratio can be computed as

$$
\eta_{B}=\frac{405 \Gamma_{\mathrm{sph}}}{4 \pi^{2} v_{w} g_{*} T} \int_{-\infty}^{\infty} d z \mu_{\tau} f_{\mathrm{sph}} e^{-\nu z}
$$

where

$$
f_{\mathrm{sph}}=\min \left(1, \frac{2.4 T}{\Gamma_{\mathrm{sph}}} e^{-40 v(z) / T}\right)
$$

modulates the baryon violation rate to account for the local Higgs field VEV [4] and $\nu=45 \Gamma_{\mathrm{sph}} /\left(4 v_{w}\right)$ accounts for washout of the baryon asymmetry in front of the wall, if it is very slowly moving. The sphaleron rate is $\Gamma_{\mathrm{sph}}=1.0 \times$ $10^{-6} T$. Figure 5 shows the differential asymmetry generated around the wall corresponding to Eq. (18) for our benchmark model.

The computation of the wall velocity $v_{w}$ is difficult $[51,52]$ and beyond the scope of this preliminary study, but for typical values $v_{w} \sim 0.1$ our predictions are rather insensitive to it since the $1 / v_{w}$ factor in (18) is largely canceled by the $v_{w}$ prefactor in the source term (17). This is evident in Fig. 6, where we show the dependence of the baryon asymmetry on $v_{w}$ for the benchmark model from

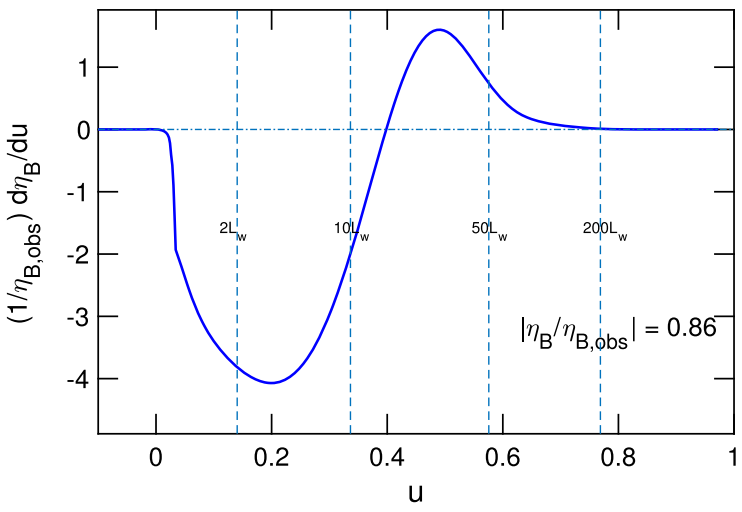

FIG. 5. The differential baryon asymmetry versus distance from the wall, $\mathrm{d}\left(\eta_{B} / \eta_{\text {obs }}\right) / \mathrm{d} u$ for our benchmark model, where $u$ is a nonlinearly rescaled variable designed to optimize the grid used for solving the fluid equations via relaxation. The center of the wall $(z=0)$ is at $u=0$ and $z \rightarrow \pm \infty$ as $u \rightarrow \pm 1$. Some physical distances are indicated by dashed lines in units of the wall thickness $L_{w}$.

Table I. As expected, it goes to zero both for very small $v_{w} \sim 10^{-4}$ [due to the $e^{-\nu z}$ factor in Eq. (18)] and at very large $v_{w}$, close to the sound velocity $1 / \sqrt{3}$, where the wall becomes a detonation, and baryogenesis is suppressed by the inability of particles to diffuse away from the wall. Between these extremes, there is a wide plateau at $v_{w} \sim 0.1-0.4$, where $\eta_{B}$ is only mildly sensitive to $v_{w}$ and it turns out that the situation is the same for other parameter sets as well. This is what motivated our assumed value for $v_{w}$.

A concern for any scenario relying upon very strongly first-order transitions is that they tend to lead to faster moving walls. Reference [53] has studied this in the context of two-field transitions such as we utilize and shown that $v_{w}$ is a monotonically increasing function of $v_{n} / T_{n}$, the strength of the phase transition at the nucleation temperature. However the exact value depends upon many parameters, only a few of which are covered in Ref. [53]. Most importantly $v_{w}$ strongly depends upon the friction of the wall due to its interactions with particles in the plasma. Our model has significant new sources of
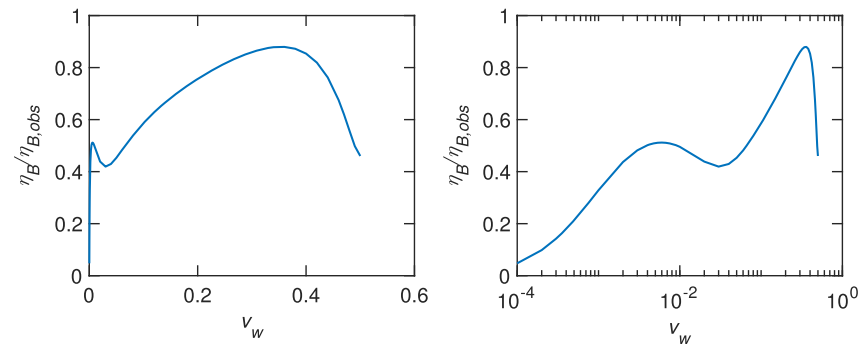

FIG. 6. The baryon asymmetry as a function of the wall velocity $v_{w}$ for our benchmark model (see Table I). The right plot is the same as the left one, but using a logarithmic scale. 
TABLE I. (First line) Parameters for a benchmark model for successful baryogenesis and observed DM abundance. (Lower lines) Mean and standard deviation of parameters from 600 random models with BAU and DM relic density of the right order of magnitude. Units are $\mathrm{GeV}$ for all dimensionful parameters. Subscript $n$ refers to quantities at the nucleation temperature.

\begin{tabular}{|c|c|c|c|c|c|c|c|c|c|c|c|c|c|c|c|c|}
\hline & $m_{\chi}$ & $m_{\phi}$ & $\lambda_{m}$ & $y$ & $|\eta|$ & $m_{s}$ & $w_{c}$ & $w_{n}$ & $v_{c}$ & $v_{n}$ & $T_{c}$ & $T_{n}$ & $v_{n} / T_{n}$ & $L_{w} T_{n}$ & $\left|\eta_{B}\right| / \eta_{\mathrm{B}, \mathrm{obs}}$ & $\Omega_{\mathrm{dm}} h^{2}$ \\
\hline Example & 55.7 & 122.4 & 0.68 & 0.66 & 0.42 & 132.2 & 91.1 & 117.7 & 79.7 & 125.9 & 127.9 & 116.1 & 1.08 & 6.8 & 0.85 & 0.11 \\
\hline Average & 50.5 & 117.0 & 0.68 & 0.56 & 0.40 & 129.4 & 98.8 & 117.1 & 94.2 & 133.0 & 124.7 & 113.5 & 1.18 & 5.9 & 1.32 & 0.36 \\
\hline Std. dev. & 5.3 & 10.3 & 0.12 & 0.14 & 0.17 & 12.6 & 10.2 & 12.2 & 9.4 & 10.8 & 2.3 & 4.2 & 0.15 & 0.8 & 2.13 & 0.39 \\
\hline
\end{tabular}

such friction with sizable couplings, through the $y \bar{L}_{\tau} \phi \chi$ and $i \eta \bar{\chi} \gamma_{5} S \eta$ interactions. We leave a more detailed investigation of $v_{w}$ in this model for future study.

\section{COLLIDER CONSTRAINTS}

The main particle physics constraint on our model is from the Drell-Yan production of the charged Higgs bosons from the inert doublet $\phi$, followed by their decay into $\tau$ and $\chi$, i.e., missing energy. This is the same signature as from pair production of $\tilde{\tau}$ sleptons in the MSSM, so we can directly apply such limits, since the production cross sections for $\tilde{\tau}_{L}$ pairs is the same as for $\phi^{ \pm}$. ATLAS has set limits from Run 1 which are not yet very constraining [54]. Only for $m_{\chi} \lesssim$ $20 \mathrm{GeV}$ and $m_{\phi}<130 \mathrm{GeV}$ is the model excluded, while for $m_{\chi} \cong 40 \mathrm{GeV}$ and $m_{\phi}<170 \mathrm{GeV}$, the allowed production cross section is less than a factor of 2 greater than the predicted one. CMS limits from Run 1 are comparable [55]. For our benchmark values we have chosen $m_{\chi} \cong 50 \mathrm{GeV}$, $m_{\phi} \cong 120 \mathrm{GeV}$, which should be probed during Run 2 of the LHC. Related analyses from Run 2 [56,57] focus on pair production of charginos decaying to $\tilde{\tau}$ rather than direct production of $\tilde{\tau}$ and so are not directly applicable to our model.

Stau searches at LEP have ruled out lighter values of $m_{\phi} \lesssim 90 \mathrm{GeV}$ for $m_{\chi} \lesssim 80 \mathrm{GeV}$ [58]. We avoid this region by restricting $m_{\phi}>100 \mathrm{GeV}$ in our scans.

Although we work primarily in the limit of no mixing of $S$ with the Higgs boson, it was pointed out that this cannot be exactly true. In the presence of small mixing $\theta_{h s}$, even though the decay channel $h \rightarrow S S$ is kinematically blocked, the invisible decay $h \rightarrow \chi \bar{\chi}$ is possible, with rate

$$
\Gamma_{\mathrm{inv}}=\frac{\eta^{2} \theta_{h s}^{2} m_{h}}{16 \pi}\left(1-\frac{4 m_{\chi}^{2}}{m_{h}^{2}}\right)^{3 / 2} .
$$

Demanding that the invisible branching ratio not exceed $30 \%$ leads to the constraint $\theta_{h s}<0.15$ for our benchmark parameters.

\section{DARK MATTER CONSTRAINTS}

\section{A. Relic density}

Because of the $Z_{2}$ symmetry under which $\phi \rightarrow-\phi$ and $\chi \rightarrow-\chi$, the lighter of the two of these particles is a stable dark matter candidate. Since $\phi$ would have a very large scattering cross section on nuclei through its weak interactions, it is preferable to assume $m_{\chi}<m_{\phi}$ so that $\chi$ is the dark matter.

The possible annihilation channels for $\chi$ are $\chi \chi \rightarrow L_{\tau} \bar{L}_{\tau}$ and $\chi \chi \rightarrow S S$. Both are $p$-wave suppressed. We find that the respective cross sections at lowest order in relative velocity are given by

$$
\begin{gathered}
\langle\sigma v\rangle_{\tau \bar{\tau}}=\frac{y^{4} m_{\chi}\left(m_{\chi}^{4}+m_{\phi}^{4}\right) T}{4 \pi\left(m_{\chi}^{2}+m_{\phi}^{2}\right)^{4}} \equiv \frac{\sigma_{0, \tau \tau}}{x}, \\
\langle\sigma v\rangle_{s s}=\frac{\eta^{4}\left(m_{\chi}^{2}-m_{s}^{2}\right)^{5 / 2} T}{4 \pi\left(m_{s}^{2}-2 m_{\chi}^{2}\right)^{4}} \equiv \frac{\sigma_{0, s s}}{x} .
\end{gathered}
$$

The relative velocity is $v^{2}=\left(s-4 m_{\chi}^{2}\right) / m_{\chi}^{2}$ and its thermal average is $\left\langle v^{2}\right\rangle=6 T / m_{\chi}=6 / x$. Our scans of parameter space favor $m_{\chi}<m_{S}$ so that the $\chi \chi \rightarrow S S$ channel is blocked, hence we focus on annihilations to $\tau^{+} \tau^{-}+\bar{\nu}_{\tau} \nu_{\tau}$.

Using the analytic approximation of Ref. [59], we find a correlation between the relic density and $y$ shown in Fig. 8 . Varying parameters in the ranges (31), we find that $0.6 \lesssim y \lesssim 0.75$ can be compatible with the observed value. Also, a larger $m_{\phi}$ correlates with a larger DM abundance, as expected from Eq. (21).

\section{B. Direct detection}

In the idealized limit of $S \rightarrow-S$ symmetry that we have considered, there are no interactions of $\chi$ with nuclei at tree level. But as remarked previously, in a realistic model it is necessary to break this symmetry to some extent, to prevent the Universe from consisting of canceling domains in which the BAU has the same magnitude but opposite signs. The most natural way this could come about is if the coupling of $\chi$ to $S$ is no longer pure pseudoscalar but takes the form

$$
S \bar{\chi}\left(\eta^{\prime}+i \eta \gamma_{5}\right) \chi
$$

since a $\chi$ loop will then induce a tadpole for $S$ proportional to $\eta^{\prime}$, leading to a VEV for $S$ at zero temperature. The VEV implies a mixing of $S$ with the Higgs boson, hence interactions with nuclei, as indicated in Fig. 7 (left panel). If the mixing angle is denoted by $\theta_{h s} \ll 1$, the cross section on nucleons is 

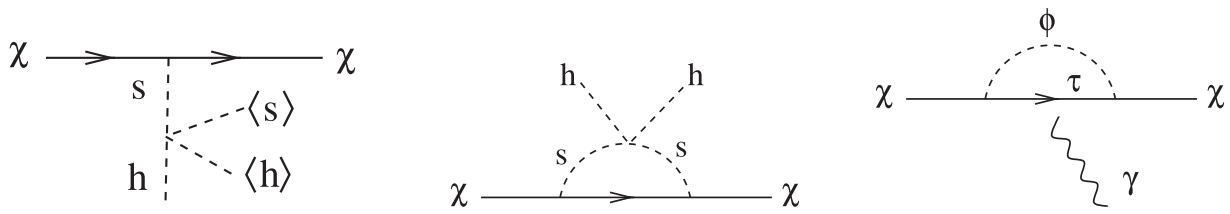

FIG. 7. Interactions contributing to dark matter-nucleon scattering. (Left panel) Higgs exchange in an extended model where $S$ mixes with Higgs. (Middle panel) Higgs exchange generated by a loop. (Right panel) Photon exchange from an anapole moment.

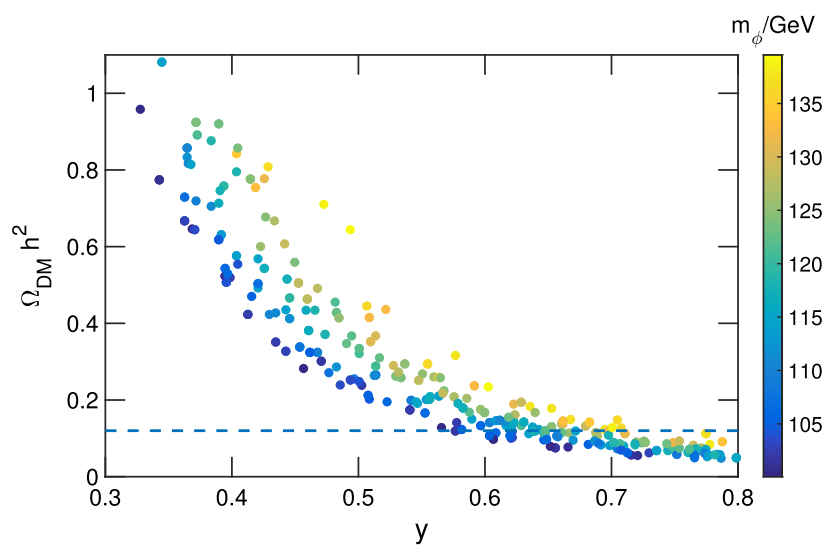

FIG. 8. Dark matter relic density versus $C P$ portal coupling $y$ from a scan of parameter space. Coloring of the dots indicates the mass of the $\phi$ particle in $\mathrm{GeV}$.

$$
\sigma_{\chi N}=\frac{1}{\pi}\left(\frac{\theta_{h s} \eta^{\prime} y_{N} \mu_{\chi N}\left(m_{h}^{2}-m_{s}^{2}\right)}{m_{h}^{2} m_{s}^{2}}\right)^{2}
$$

where $\mu_{\chi N}$ is the reduced mass and $y_{N} \cong 0.3 m_{N} / v$ is the Higgs coupling to nucleons with $v=246 \mathrm{GeV}$. ${ }^{3}$ The recent constraint from PandaX-II [60] implies

$$
\theta_{h s} \eta^{\prime}<0.04
$$

for the benchmark model of Table I (with $m_{\chi}=56 \mathrm{GeV}$, $m_{s}=109 \mathrm{GeV}$, suggesting that future direct detection may be likely for reasonable values of the parameters in this extended version of the model.

In contrast, the loop-generated interactions shown in the middle and right diagrams of Fig. 7 lead to cross sections that are much smaller. (See Ref. [61] for analysis of a similar model.) The induced Higgs portal coupling has a cross section of order

$$
\sigma \cong \frac{0.3^{2} \eta^{4} \lambda_{m}^{2} m_{\chi}^{2} m_{N}^{4}}{16^{2} \pi^{5} m_{\phi}^{4} m_{h}^{4}} \sim 10^{-52} \mathrm{~cm}^{2} .
$$

In addition to the Higgs portal, $\chi$ gets an anapole moment interaction

\footnotetext{
${ }^{3} \mathrm{We}$ omit the contribution proportional to $\eta^{2}$ which is velocity suppressed because of the $\gamma_{5}$ in the $\bar{\chi} \chi S$ vertex, which consequently gives a very small cross section $\sigma_{\chi N} \sim 10^{-51} \mathrm{~cm}^{2}$ (taking $m_{\chi} \sim 50 \mathrm{GeV}$ and $m_{S} \sim 100 \mathrm{GeV}$ ), well below the current LUX bound, even for a large mixing angle.
}

$$
\frac{e}{\Lambda^{2}} \bar{\chi} \gamma_{\mu} \gamma_{5} \chi \partial_{\nu} F^{\mu \nu}
$$

at one loop, with $\phi$ and $L_{\tau}$ in the loop. This leads to a velocitysuppressed cross section for scattering on protons [62],

$$
\sigma_{p}=\frac{e^{4}}{2 \pi \Lambda^{4}} \mu_{\chi p}^{2} v^{2}\left(1+2 \frac{\mu_{\chi p}^{2}}{m_{p}^{2}}\right),
$$

where $\mu_{\chi p} \cong m_{p}$ is the reduced mass. Estimating $\Lambda=$ $4 \pi m_{\phi} / y \sim 2 \mathrm{TeV}$ from the loop, using our fiducial parameter values, we get a cross section of order $10^{-49} \mathrm{~cm}^{2}$, still far below current sensitivities.

\section{Indirect detection}

Although the annihilations $\chi \bar{\chi} \rightarrow \tau^{+} \tau^{-}$do not lead to appreciable astrophysical signals, since they are $p$-wave suppressed, there are two associated processes that are not obviously innocuous. The one-loop diagram with virtual $\tau$ in the loop connected to two photons leads to a monochromatic line from $\chi \bar{\chi} \rightarrow \gamma \gamma$. Such lines are constrained by Fermi/LAT [63], requiring a cross section below $10^{-29} \mathrm{~cm}^{3} / \mathrm{s}$ for $m_{\chi} \sim 50 \mathrm{GeV}$, and the most cuspy assumed galactic dark matter density profile. Using Ref. [64], we find that the predicted cross section is

$$
\langle\sigma v\rangle_{\gamma \gamma} \cong 0.2 \frac{\alpha^{2} y^{4}}{256 \pi^{5} m_{\chi}^{2}} \cong 4 \times 10^{-30} \mathrm{~cm}^{3} / \mathrm{s}
$$

taking values from the benchmark model, which is still below the least conservative of the Fermi constraints.

The full cross section for $\chi \bar{\chi} \rightarrow \tau^{+} \tau^{-}$includes an $s$-wave contribution that is however helicity suppressed by $\left(m_{\tau} / m_{\chi}\right)^{2}$, which we have neglected. It was pointed out in Ref. [65] that this suppression can be overcome by internal bremsstrahlung, where a photon is emitted by the charged particle exchanged in the $t$ channel $\left(\phi^{+}\right.$in our case). Using their results, we find a cross section for emission of $\tau^{+} \tau_{-} \gamma$,

$$
\langle\sigma v\rangle_{\tau^{+} \tau_{-} \gamma} \cong 3 \times 10^{-30} \mathrm{~cm}^{3} / \mathrm{s} .
$$

Since the photon is not monochromatic, we compare the prediction to constraints on annihilation to general final states from Fermi/LAT observations of dwarf galaxies [66]. At $m_{\chi} \sim 50 \mathrm{GeV}$, the limits are of order $10^{-26} \mathrm{~cm}^{3} / \mathrm{s}$, rendering this channel harmless. The line searches thus remain the most promising avenue for indirect discovery. 


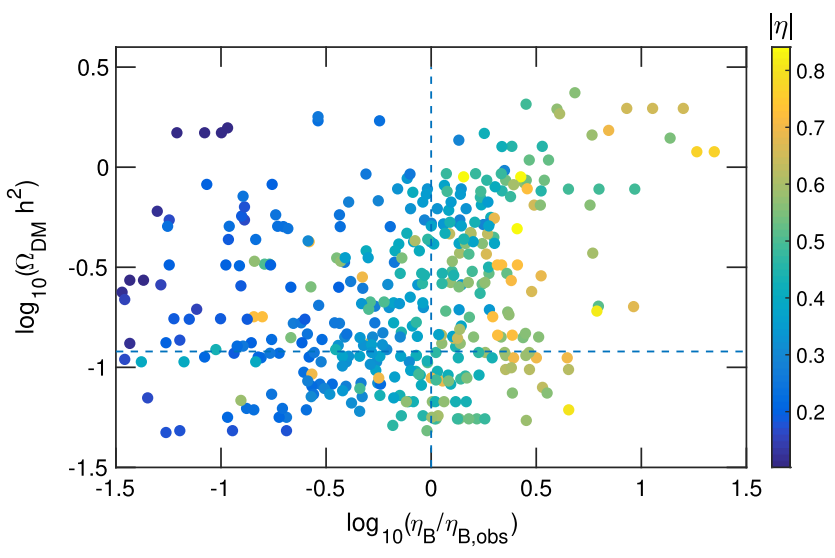

FIG. 9. Relic density versus baryon asymmetry from a scan of parameter space. Coloring of the dots shows the value of the coupling $|\eta|$.

\section{RESULTS}

We performed random scans over the model parameters to identify regions that give approximately the correct baryon asymmetry as well as the dark matter abundance. A suitable region of parameter space is given by

$$
\begin{aligned}
y & \in[0.3,0.8], \quad \eta \in[0.1,0.9], \quad \lambda_{m} \in[0.5,0.8], \\
m_{\chi} & \in[40,60], \quad m_{\phi} \in[100,140], \\
\log _{10}\left(v_{c} / w_{c}\right) & \in[-2,1.5], \quad v_{0} / v_{c} \in[1.1,10] .
\end{aligned}
$$

In a random scan over 670,000 such models, with a flat prior on the intervals (31) we find 600 examples with a strong enough phase transition, and a BAU roughly within an order of magnitude of the required value. A sample model, average values, and standard deviations ${ }^{4}$ of parameters are given in Table I. We do not claim that this is the only region of parameter space that is viable, nor do we attach any rigorous meaning to the statistics; rather our aim in this study is to demonstrate the existence of one such region, and to establish that it is not the result of any special fine-tuning of parameters.

Figure 9 plots the scatter in the plane of relic density versus baryon asymmetry, showing that it is easy to cover the target region of observed values. Moreover, out of the 600 models displayed, 21 (93) have both BAU and DM within $20 \%$ (50\%) of the observed value. The coloring of the dots shows the absolute value of the $S \bar{\chi} \chi$ coupling, which controls the size of the $C P$ violation in the model. As expected, the large asymmetry is strongly correlated with a large $|\eta|$.

In the left panel of Fig. 10 we show the correlation between baryon asymmetry and the wall width. There is only a slight positive correlation between large BAU and

\footnotetext{
${ }^{4}$ The resulting distributions are not necessarily Gaussian or symmetric around the mean values.
}
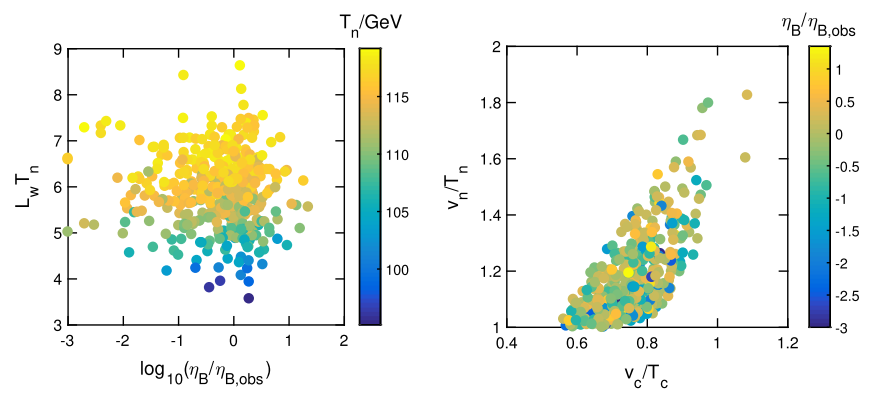

FIG. 10. (Left panel) Wall width versus the baryon asymmetry. Coloring shows the nucleation temperature in GeV. (Right panel) $v_{n} / T_{n}$ as a function of $v_{c} / T_{c}$. Coloring shows the ratio of predicted and observed baryon asymmetry.

small wall width. At any rate all wall widths are large enough for the semiclassical method used to solve the fluid equations to be valid. The right panel of Fig. 10 shows the correlation between $v / T$ evaluated at the critical and nucleation temperatures. The supercooling is never excessive; most of the models have $v_{n} / T_{n}$ between 1 and 2 . However some amount the supercooling is essential: none of the models would have survived the naive sphaleron bound $v_{c} / T_{c}>1$.

\section{CONCLUSIONS}

We have demonstrated a new class of models for electroweak baryogenesis that takes advantage of a treelevel barrier facilitated by a singlet scalar field to get a strong first-order phase transition. Unlike previous studies, we obtain the $C P$ violation needed for baryogenesis from renormalizable interactions of the scalar with hidden sector particles, in particular with the dark matter. In this way we avoid the need for unspecified new physics at a low scale, and alleviate $C P$ constraints from searches for electric dipole moments. To our knowledge, this is the first example of a model of electroweak baryogenesis where dark matter plays an essential role in providing the initial $C P$ asymmetry. We introduce the notion of a $C P$ portal interaction to transmit the $C P$ asymmetry created in the dark sector to standard model particles, as needed to bias sphalerons to produce the baryon asymmetry. This requires new particle content (an inert Higgs doublet that can decay into dark matter and leptons), which is near the discovery potential of LHC. In the present model, the $C P$ portal coupling also determines the dark matter relic density.

In this preliminary study, we have computed the baryon asymmetry quantitatively, while making a number of simplifying assumptions that could be relaxed in future work. The VEV of the scalar $S$ was taken to vanish in the true vacuum, but as noted above, there should be at least a small VEV to avoid domains containing canceling contributions to the baryon asymmetry; hence the $S \rightarrow-S$ symmetry in the scalar potential should be broken. It would be worthwhile to quantify under what conditions the higher-energy 
false vacua have time to be diluted away before they disappear by tunneling to the electroweak symmetry breaking vacuum. Breaking of the $S \rightarrow-S$ symmetry could also introduce explicit $C P$ violation into the coupling of $S$ to dark matter, as opposed to the purely spontaneous violation assumed here. Moreover it could lead to potentially observable signals for direct dark matter detection, as explained in Sec. VI B, and collider constraints from mixing of the Higgs with the singlet.

Further, we have ignored possible couplings of the inert Higgs doublet $\phi$ to the SM Higgs, and couplings of $\phi$ and $\chi$ to the $\mu$ and $e$ lepton doublets, which may be constrained by lepton flavor violating observables. And it would be interesting to determine and solve the renormalization group equations for the new couplings to see at how high a scale the model is valid before encountering a Landau pole.

Regarding the phase transition, a number of improvements could be made. These include a dynamical solution of the bubble wall profiles in field space, which we took to follow the minimum of the potential valley in the $(H, S)$ plane. More importantly, we have assumed a value for the bubble wall velocity, $v_{w}=0.3 c$, whereas an actual calculation would be desirable. Such determinations are notoriously difficult and are hampered by fundamental uncertainties in microscopic evaluation of the friction on the wall [67]; hence we leave this for future investigation. Finally one could improve on the determination of the elastic scattering rates by including all relevant channels. However, we already addressed the most pressing issue about the rates in Appendix B, where we pointed out that the dominant contributions are formally infrared divergent, and must be regularized by a selfconsistent determination of the thermal scattering rate for a fermion of fixed energy in the plasma.

Beyond technical improvements in the treatment of the model presented here, we believe that the general framework, involving a singlet scalar and new states connecting the scalar to standard model fermions, opens the door to building other new models of electroweak baryogenesis, possibly involving dark matter, and testable at the LHC.

\section{ACKNOWLEDGMENTS}

We thank Joshua Berger, Simon Caron-Huot, Felix Kahlhoefer, Guy Moore, and Pat Scott for the helpful discussions. We thank Jonathan Cornell for assistance with COSMOTRANSITIONS. This work was financially supported by Academy of Finland Project No. 278722 and by NSF Grant No. 1216168. Part of this work was performed at the Aspen Center for Physics, which is supported by NSF Grant No. PHY-1066293.

\section{APPENDIX A: IMPROVED THIN-WALL APPROXIMATION FOR TWO FIELDS}

We found the tunneling solutions for the action (10) by numerically solving the field equations

$$
\frac{d^{2} \phi_{i}}{d r^{2}}+\frac{2}{r} \frac{d \phi_{i}}{d r}=\frac{d V}{d \phi_{i}},
$$

where $\phi_{i}=h, s$, with boundary conditions $\left.\frac{d \phi_{i}}{d r}\right|_{r=0}=0$ and $(h, s) \stackrel{r \rightarrow \infty}{\rightarrow}\left(0, w_{T}\right)$, where $\left(0, w_{T}\right)$ is the $T$-dependent false minimum at high temperature. Numerical solution is straightforward, but takes several seconds per model, whereby it is not ideally suited to extensive scans over model parameters. Hence we initially tried using a (lowest order) semianalytic formula of Ref. [44] to approximate the nucleation action. Further investigation showed that this is a poor approximation, typically overestimating the action by a factor of 10 or more; see Fig. 11 .

We subsequently found that $S_{3} / T$ can be well approximated by the following, slightly modified version of the well-known thin-wall approximation [42]. Assume that the tunneling path follows a direct line between the two minima (this assumption is supported by full numerical solutions; see Fig. 2). This reduces the problem to a single effective field with potential $\tilde{V}(h, T)=V(h, s(h), T)$, where $s \equiv w_{T}\left(1-h / v_{T}\right)$. The thin-wall action then takes the usual form:

$$
S_{3 \mathrm{tw}}(T) \approx \frac{16 \pi}{3} \frac{\sigma(T)^{3}}{\Delta V(T)^{2}}
$$

where $\Delta V \equiv V\left(0, w_{T}, T\right)-V\left(v_{T}, 0, T\right)$ and the surface tension is defined as

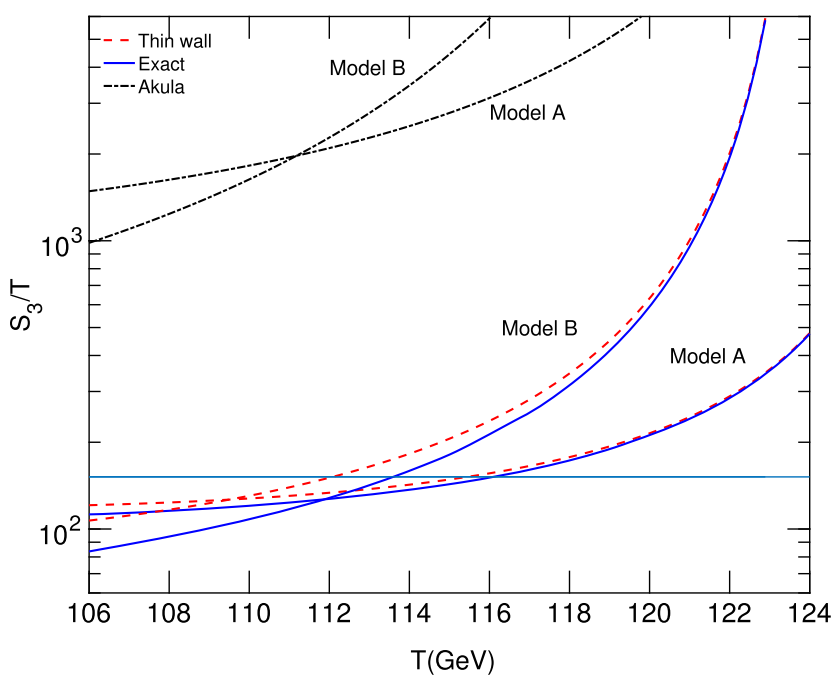

FIG. 11. Solutions for extremal action $S_{3}(T) / T$ for two different models as a function of $T$. Model A (the benchmark model) represents typical accuracy of our thin-wall approximation (red dashed lines), while model B is representative of cases where the thin-wall action deviates maximally from the exact action (blue solid lines). Black dash-dotted lines give the leading order analytic approximation from Ref. [44]. Horizontal lines (overlapping) mark the (exact) $S_{3}\left(T_{n}\right) / T_{n}$ for both models. 


$$
\sigma(T) \equiv \sqrt{1+\frac{w_{T}^{2}}{v_{T}^{2}}} \int_{0}^{v_{T}} d h \operatorname{Re}(\sqrt{2 \delta V(h, T)}),
$$

with $\delta V(h, T) \equiv \tilde{V}(h, T)-\tilde{V}(0, T)+\left(h / v_{T}\right) \Delta V(T)$. The formulas (A2)-(A3) take essentially no time to numerically evaluate and provide surprisingly accurate results, making this an ideal method for the initial model selection in an extensive parameter scan, or more generally whenever a very high precision is not needed. In Fig. 11 we show a comparison of the thin-wall and exact numerical results for some representative cases. For comparison we also show the leading order semianalytic result by Ref. [44] (black dashed-dotted curve).

\section{APPENDIX B: ELASTIC SCATTERING RATES}

Although there are many diagrams contributing to the scattering rates in Fig. 3, they turn out to be dominated by just a few. These are the diagrams with an external $\phi$, where an internal $\tau$ or $\chi$ particle in the $t$ or $u$ channel can go on shell and ones with external $\tau$ 's and $\chi$ 's where $\phi$ can go on shell in the $s$ channel. In addition the $t$ - and $u$-channel diagrams contributions are IR divergent. All these diagrams must be regularized by taking into account the thermal damping rate $\Gamma_{x}$ of the intermediate particle $x$. It amounts to using the propagator [68]

$$
\frac{i}{\not p-m_{x}+i \Gamma_{x} \not \varkappa / 2},
$$

where $u$ is the plasma 4-velocity. (This is the leading correction to the imaginary part of the self-energy from thermal corrections.) The rationalized propagator then takes the form

$$
i \frac{\not p+m_{x}}{p^{2}-m_{x}^{2}+i \epsilon},
$$

where $\epsilon=p_{0} \Gamma_{x}$. At lowest order the thermal damping rates are given by the decay rates computed in Appendix D: $\Gamma_{i}=I_{d} / n_{i}$.

Moreover, we need to subtract the on-shell contribution to avoid double counting the decays and inverse decays of $\phi$, which have already been explicitly included in the Boltzmann equations. The need for such subtractions has been discussed in similar contexts [69-71]. However there is some ambiguity in the literature as to how this should be done. A widely used prescription is to replace the squared propagator $|P(p)|^{2}$ with

$|P(p)|^{2} \rightarrow \frac{1}{\left(p^{2}-m^{2}\right)^{2}+m^{2} \Gamma^{2}}-\frac{\pi}{m \Gamma} \delta\left(p^{2}-m^{2}\right)$

in the case of a massive intermediate particle that has a decay width $\Gamma$. It has been noted [70] that this can lead to negative cross sections. Reference [71] proposes a different method of subtraction that avoids this problem, but it is specific to $s$-channel processes and does not suffice for us.

We propose to carry out a subtraction similar to (B3), but rather than doing so on the squared propagator appearing in the cross section, we apply it to the propagator in the amplitude. By construction this avoids the problem of a negative cross section and it also seems to be better justified physically. Indeed the idea is that the pole contribution gets associated with a distinct physical process: a successive decay and inverse decay of a particle, which is not part of the $2 \leftrightarrow 2$ scattering matrix but instead of the $1 \leftrightarrow 2$ matrix. It should then be removed completely, including all interference effects, which can only be ensured by subtracting at the amplitude level. For a scalar particle, our prescription is to take the principal value part of the propagator by removing the real part,

$$
P(p) \rightarrow i \frac{p^{2}-m^{2}}{\left(p^{2}-m^{2}\right)^{2}+\epsilon^{2}},
$$

where $\epsilon=m \Gamma$ for a particle with a decay width. For a fermion, we multiply (B4) by $p+m$.

The dominant contribution to the cross section for $\chi \phi$ $(\tau \phi)$ scattering, due to $t$-channel exchange, is controlled by the thermal width of $\tau(\chi)$. Similarly the cross section for $\chi \tau$ is dominated by the $s$-channel diagram controlled by the thermal width of $\phi$. Contributions from these channels are, even after pole subtractions, as large or larger than the decay contribution. That is, we are finding that the twoloop contributions to the complex part of the self-energy are very large, which means that we must solve a gap equation for the thermal widths involving all three particles and their dominant decay and scattering channels. These equations can be formally written as

$$
\Gamma_{i}=\Gamma_{d, i}+\Delta_{i}\left[\Gamma_{j}\right],
$$

where the $\Delta_{i}\left[\Gamma_{j}\right]$ 's arise from thermally averaged scattering rates:

$$
\Delta_{i}\left[\Gamma_{j}\right]=\sum_{k} n_{k}\left\langle v_{\mathrm{rel}} \sigma_{i k}\left[\Gamma_{j}\right]\right\rangle .
$$

As discussed above, we include here the dominant processes: $\chi \phi \rightarrow \chi \phi, \phi \tau \rightarrow \phi \tau$, and $\chi \tau \rightarrow \chi \tau$. In addition we included $f \tau \rightarrow f \tau$ and $\bar{f} \tau \rightarrow \bar{f} \tau$, where $f$ denotes standard model fermions. (Although these are IR convergent, taking account of the thermal mass of the $W m_{W}^{2}=(8 / 3) g^{2} T^{2}$ in $t$-channel exchange, there are many flavors.) When the dominant scattering channels are included along with the decays, the infrared sensitivity of independent channels is greatly reduced, and results stabilize against adding new contributions. For example adding $f \tau$ and $\bar{f} \tau$ channels does not change the results dramatically despite their large multiplicity. 


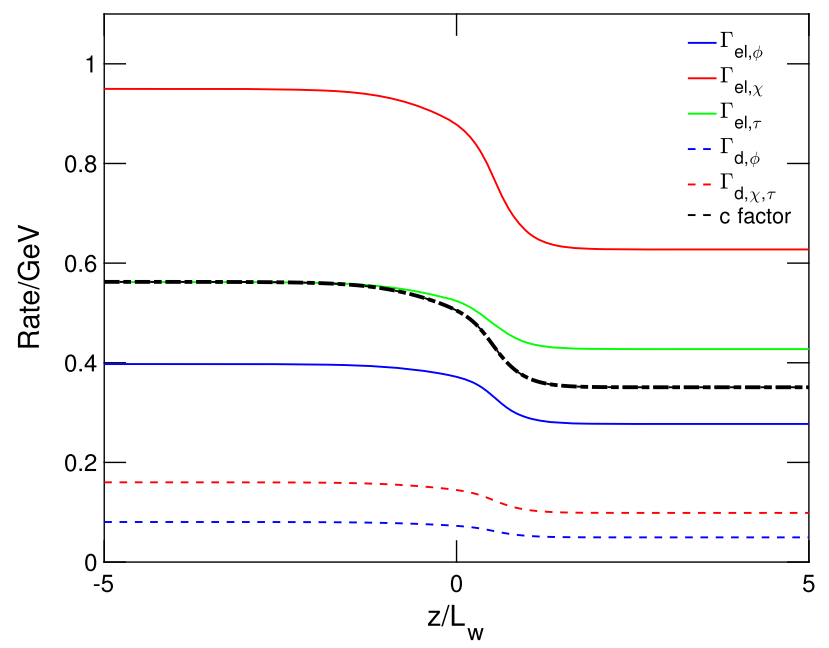

FIG. 12. Total elastic rates (solid lines) and decay rates (dashed lines) as a function of distance from bubble wall $z / L_{w}$ for our benchmark model. The decay helicity-flip $c$ factor discussed in Appendix D [Eq. (D6)] is given by the thick dashed-dotted line.

The calculation of scattering rates is straightforward and we do note show the lengthy expressions for the cross sections (computed using FEYNCALC [72]) here. We determined all thermal averages using

$$
\left\langle v_{\mathrm{rel}} \sigma_{i k}\right\rangle=\int_{\left(m_{i}+m_{k}\right)^{2}}^{\infty} \frac{\sqrt{s} \lambda^{1 / 2}\left(s, m_{i}^{2}, m_{k}^{2}\right) K_{1}\left(\frac{\sqrt{s}}{T}\right) v_{\mathrm{rel}} \sigma_{i k}}{16 T m_{i}^{2} m_{k}^{2} K_{2}\left(\frac{m_{i}}{T}\right) K_{2}\left(\frac{m_{k}}{T}\right)} \mathrm{d} s .
$$

The behavior of the solutions of Eq. (B5) in the bubble wall, along with the decay rates $\Gamma_{d, i}$, is shown in Fig. 12 for our benchmark model. Note that diffusion properties change significantly from one phase to another.

There are several other scattering channels that have IR enhancement, but are still subdominant. These are $\chi \chi \rightarrow$ $S^{*} \rightarrow \chi \chi$ annihilation, if $m_{\chi}<m_{S} / 2$ with the possibility of an $s$-channel resonance; similarly $L_{\tau} \bar{L}_{\tau} \rightarrow W^{*} \rightarrow L_{\tau} \bar{L}_{\tau}$, since the thermal mass of the $W$ in the symmetric phase is more than 2 times greater than that of $L_{\tau}$; and $L_{\tau} W \rightarrow L_{\tau} W$, where the $W$ decays to $L_{\tau} \bar{L}_{\tau}$ followed by inverse decay, similar to the $L_{\tau} \phi \rightarrow L_{\tau} \phi$ discussed above. The first process tends to be absent in our scans, which favor $m_{\chi}>m_{S} / 2$ for getting the observed BAU and DM density, and is also suppressed by $\eta / y \ll 1$ relative to the dominant processes. The others which are proportional to $g^{2}$ turn out to be numerically small compared to the dominant $y^{2}$ processes.

\section{APPENDIX C: HELICITY-FLIP RATE}

The rate of helicity flips in scattering can be computed by insertion of the helicity projection operators

$$
\Sigma_{i}=\frac{1}{2}\left(1+\gamma_{5} \grave{s}_{i}\right)
$$

into the amplitudes involving $\chi$ scattering, where the spin vector of the $i$ th particle is $s_{i}^{\mu}=\lambda_{i}\left(\left|p_{i}\right|, E_{i} \hat{p}_{i}\right)$ with $\lambda_{i}= \pm 1$ for positive or negative helicity. Carrying this out for $\chi \phi \rightarrow \chi \phi$ scattering, we find that the largest rate is for the helicity-conserving case where $\lambda_{1}=\lambda_{4}=+1$ (labeling the incoming and outgoing $\chi$ particles by $i=1$ and 4 respectively), followed by the two helicity-flipping possibilities (which have equal cross sections), and then $\lambda_{1}=\lambda_{4}=-1$. The distinction between the first and last is due to scattering on $\phi$ as opposed to $\phi^{*}$. We show the dependence of the cross sections on energy in Fig. 13 (left panel).

Carrying out the thermal averages, the ratio of the rates for either helicity-flip process versus the dominant helicityconserving one is a function of $m_{\chi} / T$ and $m_{\phi} / T$, which to a good approximation is independent of $y$, and is plotted in Fig. 13 (right panel). For numerical purposes, we find that it can be accurately approximated (to better than $1 \%$ for the parameters of interest) by a second order polynomial in $m_{\chi}$ and $m_{\phi}$ whose coefficients depend very weakly on $y$. For our benchmark model, the total flip ratio is $8 \%$.
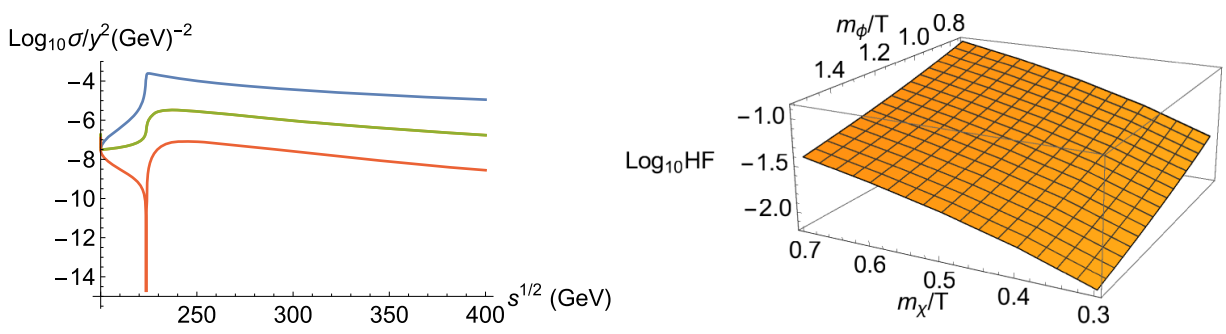

FIG. 13. (Left panel) Cross sections for helicity-conserving $\lambda_{i}=+1$ (top curve), helicity-flipping (middle curve), and helicityconserving $\lambda_{i}=-1 \chi \phi$ scattering, versus $\sqrt{s}$, for $m_{\chi}=50 \mathrm{GeV}, m_{\phi}=150 \mathrm{GeV}$, and $y=0.75$ in the thermal width of the intermediate $\tau$. (Right panel) Ratio of $\lambda_{1}=-\lambda_{4}= \pm 1$ helicity-flipping cross sections to that of the dominant $\lambda_{i}=+1$ helicity-conserving one, as a function of $m_{\phi} / T$ and $m_{\chi} / T$. It is nearly independent of $y$. 


\section{APPENDIX D: DECAY RATES}

Here we derive thermally averaged decay rates for $\phi_{ \pm} \rightarrow \chi_{h} \tau_{ \pm}$, with $h$ denoting the helicity of the $\chi$. It is straightforward to show that the collision integral in the Boltzmann equations, when expanded to first order in the chemical potentials, becomes

$$
C_{ \pm}^{h}(\mu)=\left(\mp \frac{\mu_{\phi}}{T} \pm \frac{\mu_{\tau}}{T}-h \frac{\mu_{\chi}}{T}\right) \times I_{h \pm}+\cdots,
$$

where the ellipsis represents terms that contribute to elastic integrals and

$$
\begin{aligned}
I_{h \pm}= & 2 y^{2} \int_{p} \int_{k} \int_{q}(2 \pi)^{4} \delta^{4}(p-k-q) \\
& \times\left(k \cdot q \pm M_{\chi} s_{h} \cdot k\right)\left(1-f_{0, \phi}(p)\right) f_{0, \chi}(q) f_{0, \tau}(k) \\
\equiv & I_{d} \pm h I_{h} .
\end{aligned}
$$

Here $\int_{p_{i}} \equiv \int \mathrm{d}^{3} p_{i} /\left[(2 \pi)^{3} 2 \omega_{i}\right], f_{0, i}\left(p_{i}\right)$ are the Bose-Einstein or Fermi-Dirac equilibrium distributions for $\phi, \chi$, and $\tau$, and the spin four-vector has components $s_{h}=\left(h / m_{\chi}\right) \times$ $\left(|\mathbf{q}|, \omega_{q} \hat{\mathbf{q}}\right)$. Using the Lorentz invariance of $k \cdot q$ we can reduce the first term $I_{d}$ in Eq. (D2) to a $1 \mathrm{D}$ integral,

$$
\begin{aligned}
I_{d}= & \frac{y^{2}\left(m_{\phi}^{2}-m_{\chi}^{2}\right) m_{\phi} T}{32 \pi^{3}} \int_{1}^{\infty} d u \frac{e^{m_{\phi} u / T}}{\left(e^{m_{\phi} u / T}-1\right)^{2}} . \\
& \cdot \ln \left[\frac{\cosh \left(a_{+} u+a_{-} \sqrt{u^{2}-1}\right) \cosh \left(a_{-}\left(u+\sqrt{u^{2}-1}\right)\right)}{\cosh \left(a_{+} u-a_{-} \sqrt{u^{2}-1}\right) \cosh \left(a_{-}\left(u-\sqrt{u^{2}-1}\right)\right.}\right],
\end{aligned}
$$

where $a_{ \pm}=\left(m_{\phi}^{2} \pm m_{\chi}^{2}\right) /\left(4 m_{\phi} T\right)$. It is easy to see that $\Gamma_{d} \equiv$ $I_{d} / n_{\phi}$ is the $\phi$ decay rate, which reduces to the vacuum rate in the $T \rightarrow 0$ limit. $\Gamma_{d}$ can be expressed as $y^{2} T$ times a function of $m_{\chi} / T, m_{\phi} / T$, which is shown in Fig. 14 (left panel).

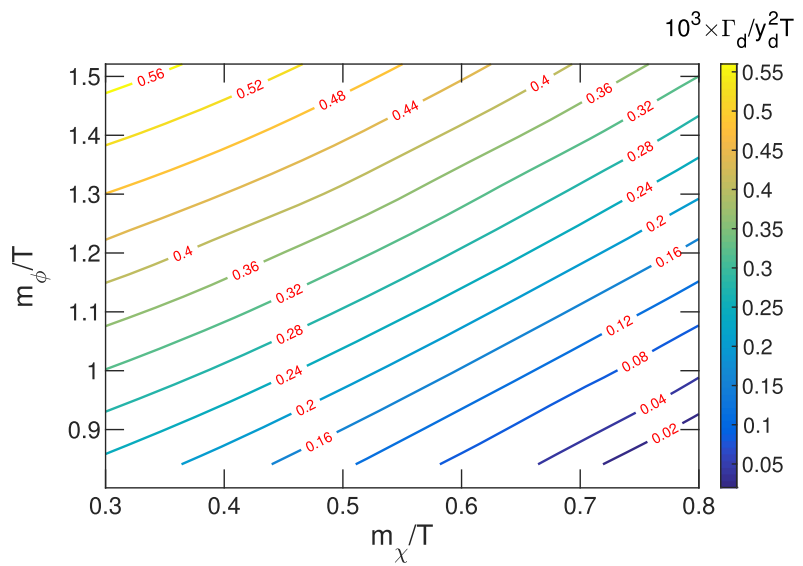

Because $s_{h} \cdot k$ is not Lorentz invariant, the second term $I_{h}$ can only be reduced to a two-dimensional integral in the most general case and we do not reproduce the result here.

These decay collision terms give rise to the following contributions to $C_{i}^{\mu}$ terms appearing in the fluid equations (14) (the superscript $\mu$ denotes the chemical potential):

$$
\begin{aligned}
\delta C_{j}^{\mu} & =\frac{1}{2 N_{j}} \sum_{h}\left(C_{+}^{h}-C_{-}^{h}\right), \quad j=\phi, \tau \\
\delta C_{\chi}^{\mu} & =\frac{g_{w}}{2 N_{\chi}} \sum_{ \pm}\left(C_{ \pm}^{+}-C_{ \pm}^{-}\right),
\end{aligned}
$$

where $N_{i}$ are the zero-mass normalization factors defined for $\chi$ below Eq. (E5). The coefficient $g_{w}=2$ in $\delta C_{\chi}^{\mu}$ is the $\mathrm{SU}(2)$ multiplicity factor.

Using Eqs. (D1) and (D2), we can write (D4) as

$$
\begin{aligned}
\delta C_{\phi}^{\mu} & =\frac{I_{d}}{T N_{\phi}}\left(\mu_{\phi}-\mu_{\tau}-c \mu_{\chi}\right), \\
\delta C_{\chi}^{\mu} & =\frac{I_{d}}{T N_{\chi}}\left(\mu_{\chi}+c \mu_{\tau}+c \mu_{\phi}\right), \\
\delta C_{\tau}^{\mu} & =\frac{2 I_{d}}{T N_{\tau}}\left(\mu_{\tau}-\mu_{\phi}+c \mu_{\chi}\right),
\end{aligned}
$$

where the helicity-flipping factor is defined as

$$
c \equiv I_{h} / I_{d} .
$$

We then infer the effective decay rates appearing in (14),

$$
\tilde{\Gamma}_{\phi}=\frac{I_{d}}{T N_{\phi}}=\frac{n_{\phi}}{T N_{\phi}} \Gamma_{\phi} \equiv r_{\phi} \Gamma_{\phi},
$$

and similarly for $\chi$ and $\tau$. The normalization factors $r_{i}$ are induced here [and in all other rates in (14)] by the particular normalization chosen for the $K_{i, j}$ functions.

The decay processes are crucial to our $C P$ portal mechanism as they are the only way to bring the $\chi C P$

FIG. 14. (Left panel) Contours of the thermally averaged, suitably scaled $\phi$ decay rate $10^{3} \Gamma_{d} / y^{2} T$ as a function of $m_{\chi} / T$ and $m_{\phi} / T$. (Right panel) Contours of the $\chi$ helicity-flip factor $c$, Eq. (D6). 
asymmetry into the $\tau$ sector. In the limit $m_{\chi} \rightarrow 0$ the $\chi$ helicity follows faithfully the chirality of $\tau$ and the transport is maximally efficient; this corresponds to the case $c=1$. However, with a nonzero $m_{\chi}$ some decays produce wrong helicity $\chi$ 's, whereby $c<1$. For a small $c$ the transport is less efficient and in the limit $c \rightarrow 0, \mu_{\chi}$ completely decouples, hiding $C P$ violation in the dark sector. We show $c$ as a function of $m_{\chi} / T, m_{\phi} / T$ in Fig. 14 (right panel): it turns out that $c$ is strongly suppressed only in the limit that $\phi$ and $\chi$ masses become degenerate, i.e., in the limit that $\chi$ is nonrelativistic (as expected).

\section{APPENDIX E: SEMICLASSICAL SOURCE IN HELICITY BASIS}

In this section we derive the semiclassical source in the helicity basis. Here we label states by their wall-frame helicities, whereas we use plasma-frame helicities in collision-term calculations. This is justified given that we calculate the BAU to first order in $v_{w}$. We start from the 3D semiclassical force derived in [50]:

$$
F_{\chi}^{s}=-\frac{m_{\chi}^{2 \prime}}{2 k_{0}}+s_{C P} \frac{s\left(m_{\chi}^{2} \theta^{\prime}\right)^{\prime}}{2 k_{0} k_{0 \|}}
$$

where $k_{0 \|}=\left(k_{0}^{2}-\mathbf{k}_{\|}^{2}\right)^{\frac{1}{2}}$ and $s_{C P}=1(-1)$ for particles (antiparticles) and $s$ refers to the spin perpendicular to the wall in the frame where the particle velocity parallel to the wall vanishes, $\mathbf{k}_{\|} \equiv 0$. That is, (E1) is the force acting on the $\mathbf{k}_{\|} \equiv 0$-frame eigenstate of the spin operator, $S_{z}=\gamma^{0} \gamma^{3} \gamma^{5}$, boosted back to the wall frame, or equivalently, it is the force acting on the eigenstates $u(p, s)$ of the boosted spin operator [50]

$$
S_{z}^{\mathrm{wf}}=\gamma_{\|}\left(S_{z}-i\left(\mathbf{v}_{\|} \times \alpha\right)_{z}\right),
$$

where the superscript wf indicates the rest frame of the bubble wall. To get the force on helicity states, we first note that we can always write a helicity state as a linear combination of $u(p, s)$ spin eigenstates: $u(p, h)=\sum_{s} c_{s} u(p, s)$. Note that force has opposite signs on opposite spins, which reflects the fact that at the quantum level $u(p, s= \pm 1)$ evolve under slightly different effective Hamiltonians. This leads to a separation of the wave function, analogously to the separation of electron spin states in the Stern-Gerlach experiment. However, our kinetic equations are classical, which by (molecular chaos) assumption forbid any correlations between initial states in collision events. This means that, consistently with the semiclassical picture, we must treat spin as a classical variable.

Thus the force acting on helicity states $u(p, h)$ is the quantum average of the force (E1) over spin $s$. In practice this means replacing $s \rightarrow\langle s\rangle_{h}$ in (E1) where $\langle s\rangle_{h}$ is the expectation value of the spin in the helicity eigenstate:

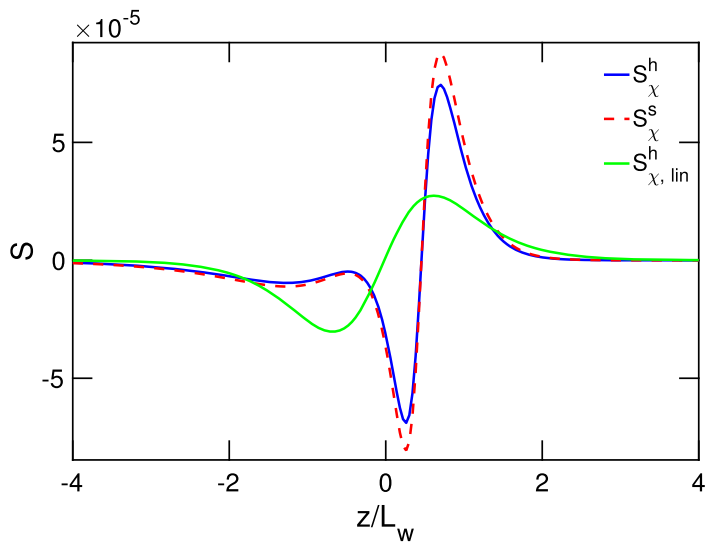

FIG. 15. The helicity source function $S_{\chi}^{h}$ used in the present work versus the spin-basis source $S_{\chi}^{s}$ of Ref. [48] (highest two curves). The lowest curve is $S_{\chi}^{h}$ computed with a straight-line approximation in $h-s$ field space to the bubble wall path, rather than the more accurate curved path.

$$
\langle s\rangle_{h}=\sum_{s} s\left|c_{s}\right|^{2}=\left\langle p, h\left|S_{z}^{\mathrm{wf}}\right| p, h\right\rangle=\gamma_{\|} \frac{h\left|k_{z}\right|}{|\mathbf{k}|},
$$

and the boost factor is $\gamma_{\|}=k_{0} / k_{0 \|}$. We can now compute the source for the fluid equations (14) arising from the force $F_{\chi}^{h}=F_{\chi}^{s=\langle s\rangle_{h}}$. Here we do not present the full calculation, performed in [48], but rather just emphasize one issue: in [48] thermal averages are defined by integrating a quantity $X$ over effective $k_{z}$ momentum, but with $k_{0}$ on shell, e.g.,

$$
\begin{aligned}
\langle X\rangle_{ \pm} \equiv & \frac{1}{N_{\chi}} \int \mathrm{d}^{4} k X\left(k_{0} ; k_{i}\right) \theta\left( \pm k_{0}\right) \\
& \times\left|2 k_{0}\right| \delta\left(k_{0}^{2}-\omega_{0}^{2}+\langle s\rangle_{h} \frac{m_{\chi}^{2} \theta^{\prime}}{k_{0 \|}}\right) \\
\approx & \frac{1}{N_{\chi}} \int \mathrm{d}^{3} k X\left(\omega_{0} \mp\langle s\rangle_{h} \frac{m_{\chi}^{2} \theta^{\prime}}{2 \omega_{0} \omega_{0 \|}} ; k_{i}\right) \\
\approx & \left\langle X\left(\omega_{0} ; k_{i}\right)\right\rangle \mp\langle s\rangle_{h} m_{\chi}^{2} \theta^{\prime}\left\langle\frac{X^{\prime}\left(\omega_{0} ; k_{i}\right)}{2 \omega_{0} \omega_{0 \|}}\right\rangle,
\end{aligned}
$$

where $N_{\chi}$ is a normalization factor, $X^{\prime} \equiv \mathrm{d} X / \mathrm{d} \omega_{0}$ and $\omega_{0}^{2}=\mathbf{k}^{2}+m_{\chi}^{2}$. This is the reason why also the $C P$-even part of the force (E1) gives rise to a $C P$-odd source (the $K_{9}^{h}$ source below). Working to leading order in gradients, one eventually finds the result (17) in the main text, where

$$
\begin{aligned}
& K_{8}^{h}(x)=\left\langle\frac{k_{z}^{2} f_{0}^{\prime}}{2 \omega_{0} \omega_{0 \|}^{2}|\mathbf{k}|}\right\rangle, \\
& K_{9}^{h}(x)=\left\langle\frac{k_{z}^{2}}{4 \omega_{0}^{2} \omega_{0 \|}^{2}|\mathbf{k}|}\left(\frac{f_{0}^{\prime}}{\omega_{0}}-f_{0}^{\prime \prime}\right)\right\rangle,
\end{aligned}
$$


with $f_{0}^{\prime}=\mathrm{d} f_{0}\left(\omega_{0}\right) / \mathrm{d} \omega_{0}$ and $N_{\gamma} \equiv \int \mathrm{d}^{3} k f_{0}^{\prime}(m=0)$. The functions $K_{8,9}^{h}$ differ from those derived from the $S_{\chi}^{s}$ source, given in [48], but in practice the difference between the helicity-basis and spin-basis sources is small. The effect is shown in Fig. 15 for our benchmark case. For comparison, we show the change in the helicity source due to adopting a straight-line approximation between the $T_{n}$ minima in the field space.
[1] S. Liebler, S. Profumo, and T. Stefaniak, Light stop mass limits from Higgs rate measurements in the MSSM: Is MSSM electroweak baryogenesis still alive after all?, J. High Energy Phys. 04 (2016) 143.

[2] M. Carena, G. Nardini, M. Quiros, and C. E. M. Wagner, MSSM electroweak baryogenesis and LHC data, J. High Energy Phys. 02 (2013) 001.

[3] D. Curtin, P. Jaiswal, and P. Meade, Excluding electroweak baryogenesis in the MSSM, J. High Energy Phys. 08 (2012) 005.

[4] J. M. Cline, K. Kainulainen, and M. Trott, Electroweak baryogenesis in two Higgs doublet models and $B$ meson anomalies, J. High Energy Phys. 11 (2011) 089.

[5] A. Haarr, A. Kvellestad, and T. C. Petersen, Disfavouring electroweak baryogenesis and a hidden Higgs in a $C P$-violating two-Higgs-doublet model, arXiv: 1611.05757.

[6] C. W. Chiang, K. Fuyuto, and E. Senaha, Electroweak baryogenesis with lepton flavor violation, Phys. Lett. B 762, 315 (2016).

[7] G. C. Dorsch, S. J. Huber, T. Konstandin, and J. M. No, A second Higgs doublet in the early Universe: Baryogenesis and gravitational waves, arXiv:1611.05874.

[8] T. Alanne, K. Kainulainen, K. Tuominen, and V. Vaskonen, Baryogenesis in the two doublet and inert singlet extension of the Standard Model, J. Cosmol. Astropart. Phys. 08 (2016) 057.

[9] S. J. Huber, T. Konstandin, T. Prokopec, and M. G. Schmidt, Electroweak phase transition and baryogenesis in the nMSSM, Nucl. Phys. B757, 172 (2006).

[10] K. Cheung, T. J. Hou, J. S. Lee, and E. Senaha, Singlinodriven electroweak baryogenesis in the next-to-MSSM, Phys. Lett. B 710, 188 (2012).

[11] W. Huang, Z. Kang, J. Shu, P. Wu, and J. M. Yang, New insights in the electroweak phase transition in the NMSSM, Phys. Rev. D 91, 025006 (2015).

[12] S. V. Demidov, D. S. Gorbunov, and D. V. Kirpichnikov, Split NMSSM with electroweak baryogenesis, J. High Energy Phys. 11 (2016) 148.

[13] J. Choi and R. R. Volkas, Real Higgs singlet and the electroweak phase transition in the standard model, Phys. Lett. B 317, 385 (1993).

[14] J. R. Espinosa, T. Konstandin, and F. Riva, Strong electroweak phase transitions in the standard model with a singlet, Nucl. Phys. B854, 592 (2012).

[15] J. R. Espinosa, B. Gripaios, T. Konstandin, and F. Riva, Electroweak baryogenesis in non-minimal composite Higgs models, J. Cosmol. Astropart. Phys. 01 (2012) 012 .
[16] J. M. Cline and K. Kainulainen, Electroweak baryogenesis and dark matter from a singlet Higgs, J. Cosmol. Astropart. Phys. 01 (2013) 012.

[17] M. Fairbairn and R. Hogan, Singlet fermionic dark matter and the electroweak phase transition, J. High Energy Phys. 09 (2013) 022.

[18] T. Li and Y. F. Zhou, Strongly first order phase transition in the singlet fermionic dark matter model after LUX, J. High Energy Phys. 07 (2014) 006.

[19] T. Alanne, K. Tuominen, and V. Vaskonen, Strong phase transition, dark matter and vacuum stability from simple hidden sectors, Nucl. Phys. B889, 692 (2014).

[20] M. Jiang, L. Bian, W. Huang, and J. Shu, Impact of a complex singlet: Electroweak baryogenesis and dark matter, Phys. Rev. D 93, 065032 (2016).

[21] F. Sannino and J. Virkajrvi, First order electroweak phase transition from (non)conformal extensions of the standard model, Phys. Rev. D 92, 045015 (2015).

[22] F. P. Huang and C. S. Li, Electroweak baryogenesis in the framework of the effective field theory, Phys. Rev. D 92 , 075014 (2015).

[23] M. L. Xiao and J.H. Yu, Electroweak baryogenesis in a scalar-assisted vectorlike fermion model, Phys. Rev. D 94, 015011 (2016).

[24] V. Vaskonen, Electroweak baryogenesis and gravitational waves from a real scalar singlet, arXiv:1611.02073.

[25] J. M. No, Large gravitational wave background signals in electroweak baryogenesis scenarios, Phys. Rev. D 84, 124025 (2011).

[26] M. Artymowski, M. Lewicki, and J. D. Wells, Gravitational wave and collider implications of electroweak baryogenesis aided by non-standard cosmology, J. High Energy Phys. 03 (2017) 066.

[27] J. Jaeckel, V. V. Khoze, and M. Spannowsky, Hearing the signals of dark sectors with gravitational wave detectors, Phys. Rev. D 94, 103519 (2016).

[28] A. Katz and A. Riotto, Baryogenesis and gravitational waves from runaway bubble collisions, J. Cosmol. Astropart. Phys. 11 (2016) 011.

[29] M. Chala, G. Nardini, and I. Sobolev, Unified explanation for dark matter and electroweak baryogenesis with direct detection and gravitational wave signatures, Phys. Rev. D 94, 055006 (2016).

[30] F. P. Huang, Y. Wan, D. G. Wang, Y. F. Cai, and X. Zhang, Hearing the echoes of electroweak baryogenesis with gravitational wave detectors, Phys. Rev. D 94, 041702 (2016).

[31] A. Beniwal, M. Lewicki, J. D. Wells, M. White, and A. G. Williams, Gravitational wave, collider and dark matter 
signals from a scalar singlet electroweak baryogenesis, arXiv:1702.06124.

[32] A. D. Dolgov, P. D. Naselsky, and I. D. Novikov, Gravitational waves, baryogenesis, and dark matter from primordial black holes, arXiv:astro-ph/0009407.

[33] J. McDonald, Electroweak baryogenesis and dark matter via a gauge singlet scalar, Phys. Lett. B 323, 339 (1994).

[34] S. Profumo, M. J. Ramsey-Musolf, and G. Shaughnessy, Singlet Higgs phenomenology and the electroweak phase transition, J. High Energy Phys. 08 (2007) 010.

[35] K. S. Babu and E. Ma, Singlet fermion dark matter and electroweak baryogenesis with radiative neutrino mass, Int. J. Mod. Phys. A 23, 1813 (2008).

[36] V. Barger, P. Langacker, M. McCaskey, M. Ramsey-Musolf, and G. Shaughnessy, Complex singlet extension of the standard model, Phys. Rev. D 79, 015018 (2009).

[37] A. Ahriche and S. Nasri, Light dark matter, light Higgs boson, and the electroweak phase transition, Phys. Rev. D 85, 093007 (2012).

[38] M. Gonderinger, H. Lim, and M. J. Ramsey-Musolf, Complex scalar singlet dark matter: Vacuum stability and phenomenology, Phys. Rev. D 86, 043511 (2012).

[39] M. Fairbairn and P. Grothaus, Baryogenesis and dark matter with vector-like fermions, J. High Energy Phys. 10 (2013) 176.

[40] S. Kanemura, N. Machida, and T. Shindou, Radiative neutrino mass, dark matter and electroweak baryogenesis from the supersymmetric gauge theory with confinement, Phys. Lett. B 738, 178 (2014).

[41] M. Lewicki, T. Rindler-Daller, and J. D. Wells, Enabling electroweak baryogenesis through dark matter, J. High Energy Phys. 06 (2016) 055.

[42] S. R. Coleman, Fate of the false vacuum: Semiclassical theory, Phys. Rev. D 15, 2929 (1977); Erratum, Phys. Rev. D 16, 1248(E) (1977).

[43] C. L. Wainwright, CosmoTransitions: Computing cosmological phase transition temperatures and bubble profiles with multiple fields, Comput. Phys. Commun. 183, 2006 (2012).

[44] S. Akula, C. Balázs, and G. A. White, Semi-analytic techniques for calculating bubble wall profiles, Eur. Phys. J. C 76, 681 (2016).

[45] G. D. Moore, Measuring the broken phase sphaleron rate nonperturbatively, Phys. Rev. D 59, 014503 (1998).

[46] K. Fuyuto and E. Senaha, Improved sphaleron decoupling condition and the Higgs coupling constants in the real singlet-extended standard model, Phys. Rev. D 90, 015015 (2014).

[47] J. M. Cline, M. Joyce, and K. Kainulainen, Supersymmetric electroweak baryogenesis, J. High Energy Phys. 07 (2000) 018.

[48] L. Fromme and S. J. Huber, Top transport in electroweak baryogenesis, J. High Energy Phys. 03 (2007) 049.

[49] K. Kainulainen, T. Prokopec, M. G. Schmidt, and S. Weinstock, First principle derivation of semiclassical force for electroweak baryogenesis, J. High Energy Phys. 06 (2001) 031.

[50] K. Kainulainen, T. Prokopec, M. G. Schmidt, and S. Weinstock, Semiclassical force for electroweak baryogenesis: Three-dimensional derivation, Phys. Rev. D 66, 043502 (2002).

[51] S. J. Huber and M. Sopena, An efficient approach to electroweak bubble velocities, arXiv:1302.1044.

[52] T. Konstandin, G. Nardini, and I. Rues, From Boltzmann equations to steady wall velocities, J. Cosmol. Astropart. Phys. 09 (2014) 028.

[53] J. Kozaczuk, Bubble expansion and the viability of singletdriven electroweak baryogenesis, J. High Energy Phys. 10 (2015) 135.

[54] G. Aad et al. (ATLAS Collaboration), Search for the direct production of charginos, neutralinos and staus in final states with at least two hadronically decaying taus and missing transverse momentum in $p p$ collisions at $\sqrt{s}=$ $8 \mathrm{TeV}$ with the ATLAS detector, J. High Energy Phys. 10 (2014) 096.

[55] V. Khachatryan et al. (CMS Collaboration), Search for electroweak production of charginos in final states with two $\tau$ leptons in $p p$ collisions at $\sqrt{s}=8 \mathrm{TeV}$, J. High Energy Phys. 04 (2017) 018.

[56] ATLAS Collaboration, Report No. ATLAS-CONF-2016093, 2016.

[57] ATLAS Collaboration, Report No. ATLAS-CONF-2016096, 2016.

[58] See http://lepsusy.web.cern.ch/lepsusy/www/sleptons_ summer04/slep_final.html; ALEPH Collaboration, Search for scalar leptons in $\mathrm{e}^{+} \mathrm{e}^{-}$collisions at centre-of-mass energies up to $209 \mathrm{GeV}$, Phys. Lett. B 526, 206 (2002); DELPHI Collaboration, Searches for supersymmetric particles in $\mathrm{e}^{+} \mathrm{e}^{-}$collisions up to $208 \mathrm{GeV}$ and interpretation of the results within the MSSM, Eur. Phys. J. C 31, 421 (2003); L3 Collaboration, Search for scalar leptons and scalar quarks at LEP, Phys. Lett. B 580, 37 (2004); OPAL Collaboration, Search for anomalous production of dilepton events with missing transverse momentum in $e^{+} e^{-}$ collisions at $\sqrt{s}=183-209 \mathrm{MeV}$, Eur. Phys. J. C 32, 453 (2004).

[59] E. W. Kolb and M. S. Turner, The early Universe, Front. Phys. 69, 1 (1990).

[60] Y. Yang (for the PandaX-II Collaboration), Search for dark matter from the first data of the PandaX-II experiment, Proc. Sci., ICHEP2016 (2016) 224 [arXiv:1612.01223].

[61] W. Chao, H.-K. Guo, and H.-L. Li, Tau flavored dark matter and its impact on tau Yukawa coupling, J. Cosmol. Astropart. Phys. 02 (2017) 002.

[62] C. M. Ho and R. J. Scherrer, Anapole dark matter, Phys. Lett. B 722, 341 (2013).

[63] M. Ackermann et al. (Fermi-LAT Collaboration), Updated search for spectral lines from Galactic dark matter interactions with pass 8 data from the Fermi Large Area Telescope, Phys. Rev. D 91, 122002 (2015).

[64] C. Garcia-Cely and A. Rivera, General calculation of the cross section for dark matter annihilations into two photons, J. Cosmol. Astropart. Phys. 03 (2017) 054.

[65] T. Bringmann, L. Bergstrom, and J. Edsjo, New gamma-ray contributions to supersymmetric dark matter annihilation, J. High Energy Phys. 01 (2008) 049.

[66] M. Ackermann et al. (Fermi-LAT Collaboration), Searching for Dark Matter Annihilation from Milky Way Dwarf Spheroidal Galaxies with Six Years of Fermi 
Large Area Telescope Data, Phys. Rev. Lett. 115, 231301 (2015).

[67] G. D. Moore, Electroweak bubble wall friction: Analytic results, J. High Energy Phys. 03 (2000) 006.

[68] D. Notzold and G. Raffelt, Neutrino dispersion at finite temperature and density, Nucl. Phys. B307, 924 (1988).

[69] E. W. Kolb and S. Wolfram, Baryon number generation in the early Universe, Nucl. Phys. B172, 224 (1980); Erratum, Nucl. Phys. B195, 542(E) (1982).
[70] J. M. Cline, K. Kainulainen, and K. A. Olive, Protecting the primordial baryon asymmetry from erasure by sphalerons, Phys. Rev. D 49, 6394 (1994).

[71] G. F. Giudice, A. Notari, M. Raidal, A. Riotto, and A. Strumia, Towards a complete theory of thermal leptogenesis in the SM and MSSM, Nucl. Phys. B685, 89 (2004).

[72] V. Shtabovenko, R. Mertig, and F. Orellana, New developments in FeynCalc 9.0, Comput. Phys. Commun. 207, 432 (2016). 